\title{
Design and Development a Social Networks Platform for Older People
}

\author{
Chien-Lung Hsu ${ }^{1}$, Kevin C. Tseng ${ }^{2}$, Chin-Lung Tseng ${ }^{1}$, and Boo-Chen Liu ${ }^{1}$ \\ ${ }^{1}$ Dept. of Information Management, Chang Gung University \\ ${ }^{2}$ Product Design and Development Lab, Chang Gung University \\ TaoYuan, Taiwan, Republic of China \\ ktseng@pddlab.org, clhsu@mail.cgu.edu.tw, \\ returntozero@livemail.tw, apoo777@hotmail.com
}

\begin{abstract}
According to the previous clinical studies, the social contact will be influenced on the life quality and health of elders. Hence, in health care for elders, how to maintain their social relationship is of primary importance. Recently, Morris proposed a conceptual model to address social isolations in a way similar to social index which can assist the elders to perceive and maintain their own social network with others whom have been previously strong ties. However, we found some drawbacks in Morris's study in display, function, cost, etc; therefore we proposed a novel social network platform attempting to display one personal social situation by using the simply behavioral feedback from the members in his/her social network. This platform includes the following properties: Addressing different information literacy and culture gap between two generations by integrating existing communication and VOIP (Voice over IP) application software into our platform, Allowing both online and offline communication, Displaying social feedback in the users' screen directly. Finally, in our study, we will adopt the TAM (Technology Acceptance Model) to validate the user's acceptance in using our developed platform.
\end{abstract}

Keywords: Elders social engagement, social networks, information literacy, culture gap on communication.

\section{Introduction}

Nowadays, aging of population is generally a social problem. Some elder people live alone because family members moving out for the employment or marriage. If these people live without any friends or relatives to chat with and concern, they will feel lonesome. It might bring about social disengagement. In order to improve elders' social engagement, Morris purposed a method to address social isolation as social indexes and then proposed a conceptual model to transform social networks as health feedback displays. She presented a solar-inspired display to depict trends of social interaction which are measured from sensor data of phone and infrared badges associated with the elders. By doing so, elders can make themselves aware of the social relationship and eager to improve or promote the interaction between people. However, there are some problems she did not consider. 
This paper reconsidered Morris's model and found some drawbacks as follows. First, Morris did not consider different information literacy. People involved in real social networks generally might be different generations. Different generations have different information literacy. For example, elders used to rely on traditional technologies (e.g., telephone) or methods (e.g., face-to-face) to interact with others, while younger might rely on emerging technologies (e.g., instant messaging). An idea social network should reduce the gap between the elders and younger. In other words, two generations are not in the same social network or platform. In order to promote social interaction between two generations, we need younger generations to participate in social network of this platform for elders.

Second, Morris's model does not consider different culture gap on communication. In social networks, different generations have their best ways to express themselves. For example, some elders are good at face-to-face interaction, while younger aren't. Most of younger are over reliant on emerging technologies (e.g., instant messaging) which causes them not to be good at expressing their true mind by direct communication (e.g., telephone), face-to-face communication. An idea social network should reduce the gap between the elders and younger. In other words, communication barrier between two generations should be overcome.

Third, Morris's display feedbacks only considered and displayed simple social relationship. Social index is generated by the movement toward and away from the elder. The elder is only impressed by perceiving the relationship. If the display can further show some critical social information, it can help the elders to improve his sense of joy. For example, if the system can display who actively pays concern for elders, elders will know who is concerned for them. They will get higher impression from it.

In this paper, we purposed some methods to solve the above problems. First, we integrated VoIP, instant messenger into our social platform. Through our social platform, two generations can participate in the same social network. In this social network, younger can send instant message to elders, while elders can make a phone call on internet to younger. The information gap between two generations can be converged.

Second, by integrating instant messaging, younger can use instant messaging to express their true mind to elders. In that case, two generations can interact with each other without feeling embarrassed. Therefore, we overcome the communication barrier between two generations.

Third, in our display feedback, our solar system can display more metaphor messages. For example, our system can display who is actively concern for a person by the size of planets. User can know which friends are concerned for him and feel a sense of existence. The sense of existence will pleasure elderly user.

\section{Literature Review}

\subsection{Social Networks as Feedback Display}

How to display one's social networks? In 2005, Morris [1] applied principles from cognitive-behavioral and mindfulness therapy to address social isolation as social 
indexes and then proposed a conceptual model to transform social networks as health feedback displays. She presented a solar-inspired display to depict trends of social interaction for the elderly. Social activities are measured from sensor data of the phone and infrared badges associated with the elders. This means that the measures are based on the amount of interaction by face to face and telephone. By doing so, elders can make themselves aware of the social relationship and eager to improve or promote the interaction between people. The elders can then increase social interaction by interacting with family or friends and furthermore improve their quality of life and health. However, she did not consider different information literacy between two generations and different culture gap on communication. Those problems might bring less interact between family members, young members and elderly member especially.

\subsection{Different Information Literacy}

Different generations have different technology using. Zoe Roupa et al [4]. found that the result of using technology by the elderly. The research reported that elders were familiar with using TV, washing machine, vacuum, iron, cooker, wireless phone. More in detailed, women were more familiar to using washing machine, vacuum cleaner, electric iron, stove than men, while men were more familiar to using wireless phone than women. Young generation was familiar to the use of computer and internet. Young people are associated with web and internet. In other words, two generations are not in the same social network or platform. Therefore, they can't always have a same space or opportunity to communicate or share some information with each other to promote family social interaction.

\subsection{Different Culture Gap on Communication}

As the growing of the internet, more and more people are relying on internet, especially young people. On internet communication, young people can express themselves without anxiety. People who easily get social anxiety also can fluently express themselves by internet. The internet seems to have this value to help people overcome communication problems like feeling nervous reported by Tom R. Tyler(2002)[5]. However, most of young people are over reliant on emerging technologies (e.g., instant messaging) which causes them not to be good at expressing their true mind by direct communication (e.g., telephone), face-to-face communication. On the other hand, elders are good at face-to-face interaction or direct communication (e.g., telephone). In this case, two generations might have a communication problem.

\subsection{Technology Acceptance Model (TAM)}

Technology Acceptance Model is a method to explain and anticipate a user's acceptance of IT. TAM is based on a theory called Theory of Reasoned Action (TRA), which purposed by Fishbein and Ajzen. TRA suggests that an individual's behaviors are determined by personal intention. Personal intention is determined by attitude. In 1989, Davis[6] purposed TAM which suggests that perceived usefulness and perceived ease of use of IT are major factors of IT usage. The definition of perceived usefulness defined by Davis is "the degree of which a person believes that using a particular system would enhance his or her job performance." The definition of 
perceived ease of use is "degree of which a person believes that using a particular system would be free of effort."

\section{System Design and Practice}

\subsection{Social Communication Platform Practice and Design}

In this paper, we reconsidered Morris's model and purposed some methods to solve Morris's problems. In order to remove the difference of the information literacy between two generations, we integrate VoIP, instant messenger into our social platform. Moreover, we designed a friendless user interface for elders. Elders can operate the application by laptop or tablet PC easily. They could just use one finger to select a one of their family members and make a phone call shown in figures 1 and 2 .

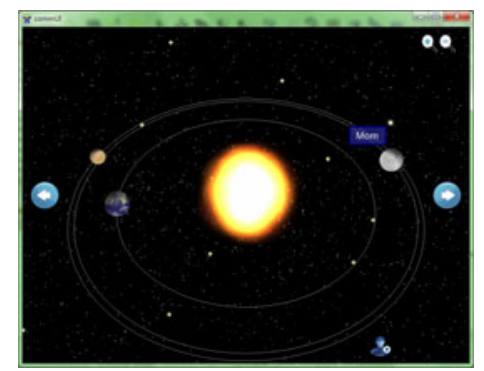

Fig. 1. Main user interface. This shows a user can pick a planet to get into a communication tools interface. A planet represents a family member or a friend. When users interact with their friend as more as possible, planets will come closer to sun. It represents that the friend is closed to you.

User needn't bother dialing phone number because every friend's contact information has been set up when user added a friend. In addition, elders can easily read messages from family members. When one of elders' family members sends a message from MSN or E-mail, elders will be notified by system shown figure 3. Therefore, through our social platform, two generations can participate in the same social network. In this social network, younger can send instant message to elders, while elders can make a phone call on internet to younger. The information gap between two generations can be converged.

Second, by integrating instant messaging, younger can use instant messaging to express their true mind to elders. In that case, two generations can interactive with each other without feeling embarrassed. Therefore, we overcome the communication barrier between two generations.

Third, in our display feedback, our solar system can display more metaphor messages. For example, our system can display who is actively concerned for a person by the size of planets shown figure 1. A planet become bigger, which represents a friend being actively paying concern with elders. Elders can know which friends 
are concerned for him and feel a sense of existence. The sense will pleasure elders. Moreover, when elders have more and more talks with their family, their screen will be full of shining stars. In that case, we expect that it can promote and eager a user to interact with friends or family members.
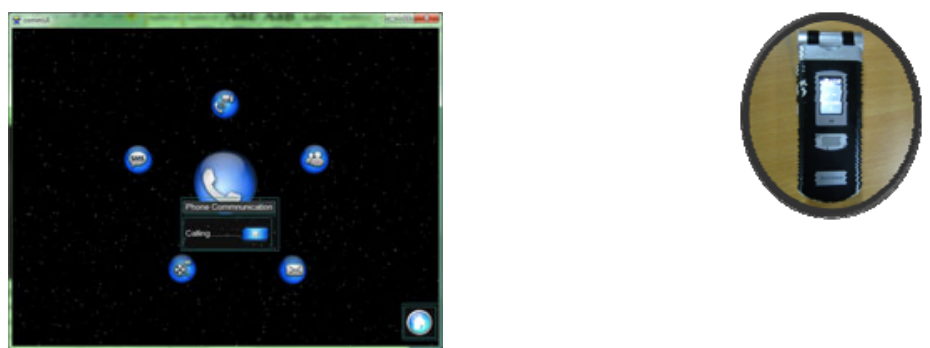

Fig. 2. Communication tools. This shows a user pick a phone button on the center of the screen. Then friend's phone you call will start ringing.

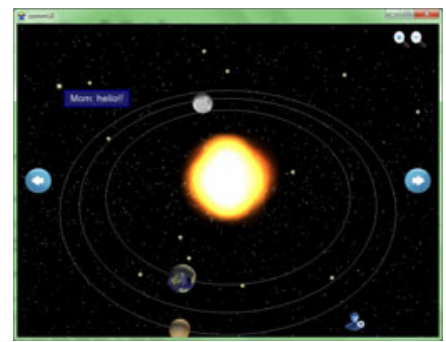

Fig. 3. Message receiving. A friend leaves a message, user can receive message by this interface. System will also notify user by showing newest message.

Table 1. The classification of communication tools

\begin{tabular}{|c|c|c|c|}
\hline \multirow{7}{*}{ Online } & \multirow{2}{*}{ Internet-Disable } & Multimedia Message & None \\
\hline & & Text Message & None \\
\hline & \multirow{5}{*}{ Internet-Enable } & \multirow{2}{*}{ Multimedia Message } & Skype phone \\
\hline & & & Skype Video phone \\
\hline & & \multirow{3}{*}{ Text Message } & Skype message \\
\hline & & & E-mail \\
\hline & & & MSN \\
\hline \multirow{6}{*}{ Offline } & \multirow{2}{*}{ Internet-Disable } & Multimedia Message & MMS \\
\hline & & Text Message & SMS \\
\hline & \multirow{4}{*}{ Internet-Enable } & \multirow{2}{*}{ Multimedia Message } & Video Message \\
\hline & & & Voice Message \\
\hline & & \multirow{2}{*}{ Text Message } & E-mail \\
\hline & & & MSN \\
\hline
\end{tabular}




\subsection{Communication Tools Selection}

Our system included several communication tools: MSM, Skype, E-mail, Video message, Voice message, SMS, MMS, etc. We chose these representative communication tools as a sample based on our model shown table 1.We defined and classified the characteristics of all communication tools. In our classification, all communications can be classified according to online or offline interaction. Online interaction and offline interaction are classified into internet-disable and internet-enable. Internet-disable and internet-enable are aslo classified into multimedia message and text message.

\section{Discussion}

In this paper, we used TAM to verify whether this system can be accepted or not by user. The model for our research shown figure 4 is an extension of the TAM. Perceived friendliness, the extended part of TAM, is construct of feeling concerned because it brings the question of how seeing social concerned feedback display affect the individual's acceptance of our social platform. The assumption is that perceived friendliness will have a positive effect on the individuals' attitude toward using our social platform and users' behavioral intentions to use our social platform. Feeling concerned from others, we call it Perceived Friendliness, is necessary in our system. In social platform, feeling concerned is an important factor of effect on attitude toward using social platform.

Therefore we state Hypothesis 1. Perceived Friendliness will be a positive relationship to Attitude toward Using. Perceived ease of use and perceived usefulness is the basic of TAM. We also verify the following hypothesis.

Hypothesis 2. Perceived Ease of Use will be a positive relationship to Attitude toward Using.

Hypothesis 3. Perceived Usefulness will be a positive relationship to attitude toward Using.

Hypothesis 4. Perceived usefulness will be a positive relationship to Behavioral Intention to Use.

Hypothesis 5. Attitude toward Using will be a positive relationship to Behavioral Intention to Use.

\subsection{Sample}

The sample consisted of 32 students aged 20 to 24 , who were majoring information management. The data were gathered by means of questionnaire. 32 usable questionnaires were used for analysis.

\subsection{Internal Consistency Reliability}

The coefficients are represented for each of the constructs in Table 2 . The values of reliability range from 0.64 (for perceived ease of use) to 0.90 (for Behavioral intentions to use). According to the exploratory nature of the study, reliability of the scales was deemed adequate. 
Table 2. Consistency Reliability

\begin{tabular}{|l|l|l|l|l|}
\hline Constructs & Definition & Mean & Standard Deviation & Reliability \\
\hline PEOU & Perceived ease of use & 3.92 & 0.57 & 0.64 \\
\hline PF & Perceived friendliness & 4.03 & 0.62 & 0.84 \\
\hline PU & Perceived usefulness & 3.92 & 0.61 & 0.76 \\
\hline ATT & Attitude toward using & 4.02 & 0.46 & 0.68 \\
\hline BIU & Behavioral intentions to use & 3.88 & 0.64 & 0.90 \\
\hline
\end{tabular}

\subsection{Construct Validity}

Table 3 shows that the result of the factor analysis with varimax rotation. Five factors were extracted.

Table 3. Scale factorial validity

\begin{tabular}{|c|c|c|c|c|c|}
\hline Scale items $^{\text {a }}$ & \multicolumn{5}{|c|}{ Factors } \\
\cline { 2 - 6 } & $\mathbf{1}$ & $\mathbf{2}$ & $\mathbf{3}$ & $\mathbf{4}$ & $\mathbf{5}$ \\
\hline ATT1 & -.106 & .392 & .155 & .679 & .331 \\
ATT2 & .399 & .458 & .055 & .576 & -.156 \\
ATT3 & .223 & -.112 & -.002 & .836 & .104 \\
BIU1 & .740 & .262 & .068 & .217 & .169 \\
BIU2 & .928 & .075 & .175 & .099 & .045 \\
BIU3 & .835 & .185 & .272 & .111 & -.071 \\
BIU4 & .720 & .183 & .137 & .074 & .197 \\
PEOU1 & .205 & -.015 & .005 & .007 & .809 \\
PEOU2 & -.013 & .281 & .008 & .223 & .836 \\
PF1 & .341 & .820 & .077 & -.059 & .157 \\
PF2 & .052 & .891 & .153 & .163 & .182 \\
PF3 & .550 & .634 & .069 & .113 & -.064 \\
PU1 & .099 & .158 & .845 & .187 & -.016 \\
PU2 & .549 & .103 & .738 & -.100 & -.047 \\
PU3 & .541 & .009 & .551 & -.060 & .168 \\
\hline
\end{tabular}

${ }^{a}$ ATT1-ATT3, attitude toward using; PL1-PL3, perceived friendliness items; BIU1-BIU3, behavioral intention to use; PEOU1-PEOU2. perceived ease of use items; PU1-PU3, perceived usefulness items; $\mathrm{n}=32$.

\subsection{Analysis Results}

The hypothesized relationships were tested using regression analysis. Table 4 shows a result of the hypothesis test.

Hypothesis 1 examines the relationship between the perceived friendliness and attitude toward using: perceived friendliness is significantly related with attitude toward using $(\beta=0.372$, $\mathrm{p}$-value $=0.055, \mathrm{p}<0.1)$. Hypothesis 1 was supported.

Hypothesis 2 examines the relationship between perceived ease of use and attitude toward using: perceived ease of use is insignificantly related with attitude toward using $(\beta=0.193$, $p$-value $=0.269, p>0.1)$. Hypothesis 2 was not supported. This result said that users might perceive ease of use, but users did not think the ease of use can change their attitude toward using our system. 
Hypothesis 3 examines the relationship between perceived usefulness and attitude toward using $(\beta=0.084$, $p$-value $=0.640, p>0.1)$. Hypothesis 3 was not supported. This result said that users might perceive system usefulness, but users did not think the usefulness of system can change their attitude toward using system.

Hypothesis 4 examines the relationship between perceived usefulness and behavioral intentions to use $(\beta=0.526$, $\mathrm{p}$-value $=0.001, \mathrm{p}<0.001)$. Hypothesis 4 was supported.

Hypothesis 5 examines the relationship between attitude toward using and behavioral intentions to use $(\beta=0.272$, $\mathrm{p}$-value $=0.072, \mathrm{p}<0.1)$. Hypothesis 5 was supported.

Table 4. Results of hypotheses tests

\begin{tabular}{|l|l|l|l|l|}
\hline $\begin{array}{l}\text { Dependent Variable } \\
\text { a }\end{array}$ & $\begin{array}{l}\text { Independent } \\
\text { Variable }^{\mathbf{a}}\end{array}$ & Beta & P-value & Hypothesis result \\
\hline ATT $\left(\mathbf{R}^{\mathbf{2}}=\mathbf{0 . 1 7 5}\right)$ & PEOU & 0.193 & 0.269 & H2 was not supported \\
& PU & 0.084 & 0.640 & H3 was not supported \\
\cline { 2 - 5 } & PF & $0.372 *$ & 0.055 & H1 was supported \\
\hline BIU $\left(\mathbf{R}^{\mathbf{2}}=\mathbf{0 . 3 8 2}\right)$ & ATT & $0.272^{*}$ & 0.072 & H5 was supported \\
& PU & $0.526 * * *$ & 0.001 & H4 was supported \\
\hline
\end{tabular}

$* \mathrm{p}<0.1 ; * * \mathrm{p}<0.05 ; * * * \mathrm{p}<0.01$.

a ATT, attitude toward using; PEOU. perceived ease of use; PU, perceived usefulness; PF, perceived friendliness; BIU, behavioral intentions to use.

\section{Conclusions}

Due to the situations of aging society, we believe that the health care service for elders will become a trend in the future. According to previous clinical studies and literatures, we find the social contact will be a critical factor in determining the life

Table 5. System comparison with Morris

\begin{tabular}{|l|l|l|l|}
\hline \multicolumn{2}{|c|}{ System Model } & Morris & $\begin{array}{l}\text { Our } \\
\text { system }\end{array}$ \\
\hline \multirow{5}{*}{ Display Part } & Social distance showing on screen & 0 & 0 \\
\cline { 2 - 4 } & $\begin{array}{l}\text { Displaying who are actively concerned about } \\
\text { myself }\end{array}$ & $\mathrm{x}$ & $\circ$ \\
\hline \multirow{4}{*}{$\begin{array}{l}\text { Function } \\
\text { Part }\end{array}$} & Online Communication & $\circ$ & $\circ$ \\
\cline { 2 - 4 } & Offline Communication & $\mathrm{x}$ & $\circ$ \\
\cline { 2 - 4 } & Internet-Disable Communication & $\circ$ & $\circ$ \\
\cline { 2 - 4 } & Internet-Enable Communication & $\mathrm{x}$ & $\circ$ \\
\cline { 2 - 4 } & Text Message Communication & $\mathrm{x}$ & $\circ$ \\
\cline { 2 - 4 } & Voice Communication & $\circ$ & $\circ$ \\
\cline { 2 - 4 } & Face to Face Communication & $\circ$ & $\circ$ \\
\cline { 2 - 4 } & Media Communication & $\mathrm{x}$ & $\circ$ \\
\hline
\end{tabular}


Table 6. Benefits comparison with Morris

\begin{tabular}{|l|l|l|}
\hline \multicolumn{1}{|c|}{ Benefits } & Morris & Our \\
\hline Promote social contact & 0 & 0 \\
\hline The elderly life quality improvement & 0 & 0 \\
\hline converge information gap, promote two generations interaction & $\mathrm{x}$ & 0 \\
\hline converge interaction gap, break communication barrier & $\mathrm{x}$ & 0 \\
\hline Interacting at anytime and anywhere & $\mathrm{x}$ & 0 \\
\hline Waiting and traveling time cost & High & Low \\
\hline
\end{tabular}

quality and health for elders. In this study, we first review Morris's study and identify some drawbacks in his research. We further proposed a novel social network platform and compared some critical properties in the platform with those in Morris's as shown in Tables 5 and 6. As shown in these tables, our proposed platform provides more functional properties and benefits than those of Morris; moreover, our platform also reduces the waiting and traveling time cost.

In conclusion, our social network platform provides unique, friendly, simple, and useful functional properties. We believe it can promote the younger generation to pay more attention to the elders and to reconstruct an available communication channel between the two generations.

\section{References}

1. Morris, M.E.: Social networks as health feedback displays. IEEE Internet Computing 9, 29-37 (2005)

2. Hendryx, M., Green, C.A., Perrin, N.A.: Social support, activities, and recovery from serious mental illness: STARS study findings. Journal of Behavioral Health Services and Research 36, 320-329 (2009)

3. Moon, J.-W., Kim, Y.-G.: Extending the TAM for a World-Wide-Web context. Information \& Management 38, 217-230 (2001)

4. Roupa, Z., Nikas, M., Gerasimou, E., Zafeiri, V., Giasyrani, L., Kazitori, E., Sotiropoulou, P.: The Use of Technology by the elderly. Health Science Journal 4, 118-126 (2010)

5. Tyler, T.R.: Is the Internet Changing Social Life? It Seems the More Things Change, the More They Stay the Same. Journal of Social Issues 58, 195-205 (2002)

6. Davis Jr., F.D.: Perceived usefulness, perceived ease of use, and user acceptance of information technology. MIS Quarterly 13, 319-340 (1989)

7. Fishbein, M., Ajzen, I.: Belief, "Attitude, Intentions and Behavior: An Introduction to Theory and Research". Addison Wesley, Boston (1975)

8. Sarason, I.G., Sarason, B.R., Perce, G.R.: Social Support, Personality and Health. In: Topics in Health Psychology, pp. 245-256. John Wiley \& Sons, Chichester (1988)

9. Morris, M., et al.: New Perspectives on Ubiquitous Computing from Ethnographic Study of Elders with Cognitive Decline. In: Dey, A.K., Schmidt, A., McCarthy, J.F. (eds.) UbiComp 2003. LNCS, vol. 2864, pp. 227-242. Springer, Heidelberg (2003)

10. Frost, J., Smith, B.K.: Visualizing Health: Imagery in Diabetes Education. In: Proc. Designing for User Experience. ACM Press, New York (2003), http: / /www . aiga . org / resources/content/9/7/8/documents/frost.pdf 


\section{Appendix A}

Perceived ease of use [five-point Likert type scale]

(1) It is easy to operate the system

(2) Using the system requires a lot of mental effort

Perceived usefulness [five-point Likert type scale]

(1) Using this social system can help me to concern with those friends I forget.

(2) Using this social system can let me interact with my family member actively

(3) Using this social system can let me know the social condition with my family member

Perceived friendliness [five-point Likert type scale]

(1) Using this social system can let me know who is paying concerned with me

(2) Using this social system, I will have a sense of being concerned

(3) I will be enjoyed seeing my relationship

Attitude toward using [five-point Likert type scale]

(1) Using this system is a positive idea

(2) Using this system is a wise idea

(3) Using this system is pleasant idea

Behavioral intentions to use [five-point Likert type scale]

(1) I will use this system to control my social condition

(2) I will use this system to promote me and my family to interact

(3) I will use this system to promote me and my friends to interact

(4) I will suggest my family using this system 